\title{
Atypical radicular cyst - a case report
}

\author{
Sadeta Parusheva ${ }^{1}$, Gerard Ojeda ${ }^{2}$, Alexandra \\ Traykova $^{3}$, Dimitar Yovchev ${ }^{4}$, Elitsa Deliverska ${ }^{1}$
}

1. Department of Dental, Oral and Maxillofacial surgery, Faculty of Dental Medicine, Medical University of Sofia; 2. 5-th City Hospital, Sofia; 3. Student in Faculty of Dental Medicine, Medical UniversitySofia; 4. Department of Imaging and Oral Diagnostics, Faculty of Dental Medicine, Medical University- Sofia;

\begin{abstract}
A radicular cyst is an inflammatory odontogenic cyst. Radiographically it presents as a well-defined periapical radiolucency. We report a case of a 20-year-old female with a mixed radiographic image(CBCT), diagnosed as radicular cyst in the periapical area of the roots of the central and lateral upper right incisors. The teeth have undergone a well-performed endodontic treatment. Extraction of the upper right lateral incisor with enucleation of the cyst was performed. Intraoperatively macroscopically, a rounded formation is observed, occupying the anterior part of the palate, reaching to the area of the premolars. Microscopically, a cystic formation lined by non-keratinized stratified squamous epithelium covered with multilayered epithelium was found. A radicular cyst with bone sequestration was diagnosed. Although rare, radicular cysts should be considered in the differential diagnosis of mixed periapical lesions especially in cases of tooth with pulp necrosis and/or one that has undergone endodontic treatment.
\end{abstract}

Keywords: atypical radicular cyst, mixed radiographic image, enucleation, $\mathrm{CBCT}$, bone sequestrum 


\section{Background}

Radicular cyst is a true cyst and is a pathological cavity bounded by a connective tissue capsule lined with epithelial cells and filled with liquid content. [1] This periapical cyst is the most common odontogenic cyst and is a result of proliferation of epithelial rests of Malassez induced by inflammatory cytokines and growth factors released by inflammatory cells found in chronic apical periodontitis following root canal infection and pulp necrosis. As a result of infectious irritation, a cystic cavity filled with liquid contents is formed. These cysts develop in both jaws, with a higher frequency in the frontal area. They are found mainly in the permanent dentition. Radicular cysts develop deep in the alveolar bone and have slow, asymptomatically growing. That's why their detection is accidental during X-ray examination of non-vital teeth on another occasion. The development of cysts sometimes leads to unusual sensations such as heaviness, stretching or neuralgic pain. Mobility or tilting of affected or adjacent teeth occurs in large cystic formations. Radiographically, radicular cyst appears usually as a well-circumscribed periapical radiolucent image in a tooth with pulp necrosis or tooth with bad endodontic treatment. [2] Presence of calcifications is commonly observed in these lesions, but it is usually discrete and observed only in histopathological analysis. Radicular cyst appearing as a mixed radiographic image is very rare and review of the literature revealed several cases of mixed radiographic image diagnosed as radicular or residual cyst formations.

\section{Aim}

The purpose of this report is to present a rare case of mixed/heterogeneous image in the periapical region of endodontically treated teeth after trauma in this area.

\section{Case report}

A 20-year-old female patient was attended in a Department of Dental, Oral and Maxillofacial surgery, FDM, MU- Sofia with a swelling in the right anterior region of the maxilla palatally. The medical history was noncontributory with anamnesis of trauma 8 years ago and endodontic treatment 6 years ago.

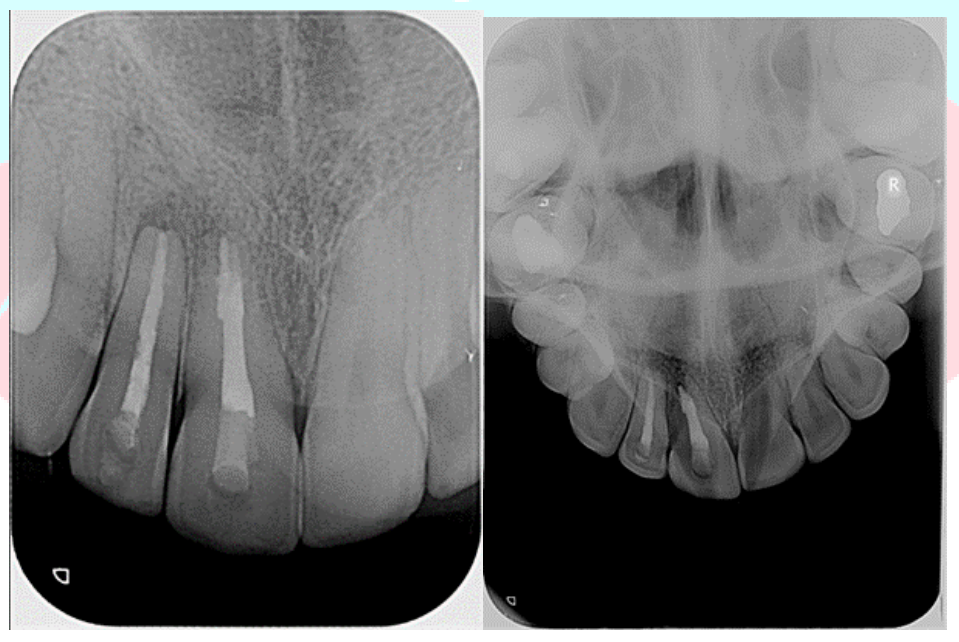

Fig. 1 X-Ray of the frontal upper region, showing periapical lesion 
In intraoral examination, a swelling palatally in the region of the maxillary right central and lateral incisors was detected. The swelling presented with firm consistency, the superficial mucosa was intact and showing normal colour. Cone beam computed tomography revealed a mixed, well-circumscribed image located in the periapical region of the maxillary right central and lateral incisors, which were with satisfactory endodontic treatment. The lesion showed a piece of hyperdense focus inside and disruption of the palatal cortical bone. (fig.1,2) Calcifying cystic odontogenic tumor, ossifying fibroma and adenomatoid odontogenic tumor were the hypotheses of differential diagnosis. Surgery under local anesthesia was scheduled.

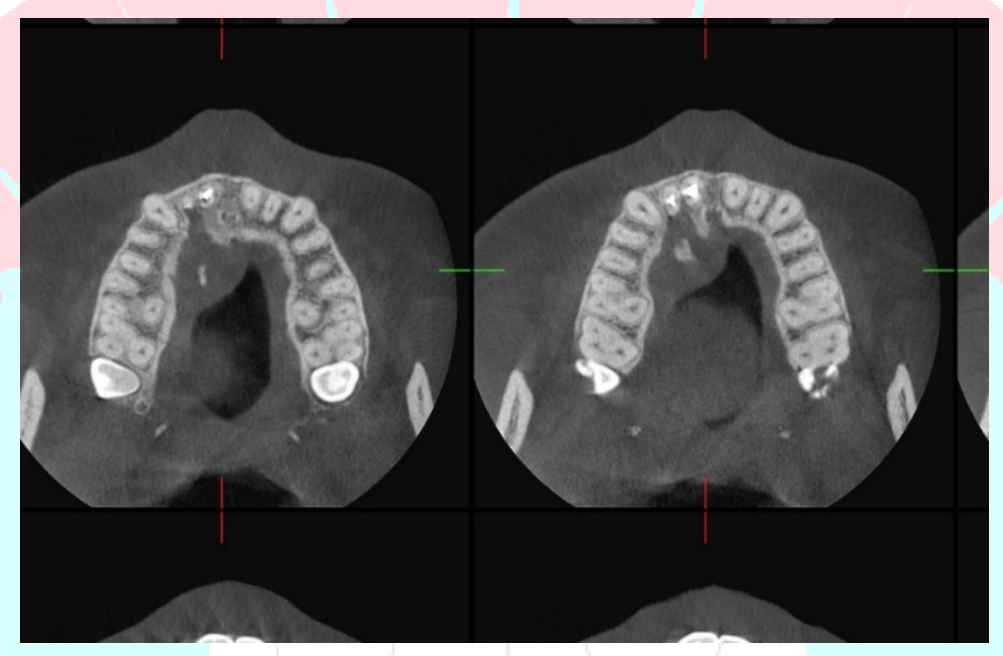

Fig. 2a. Axial plane of the upper region

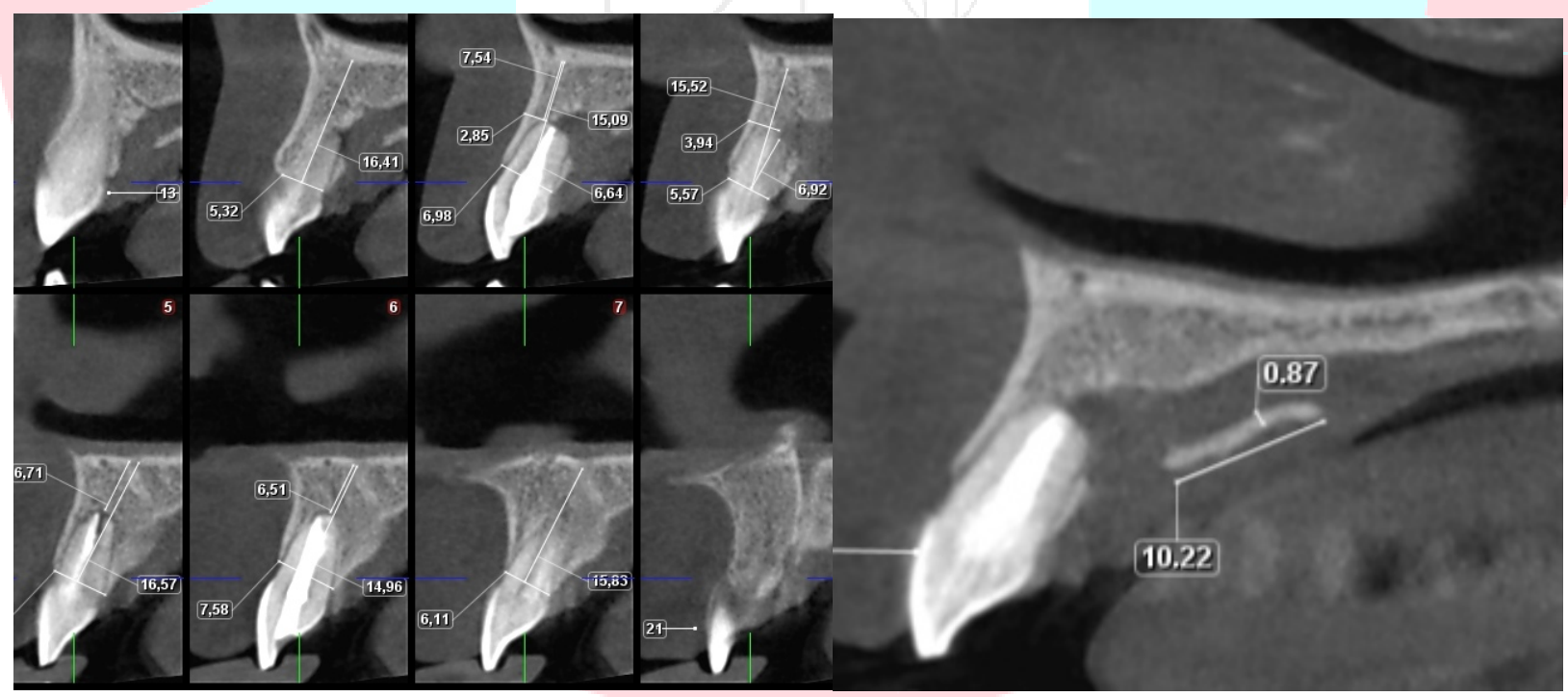

Fig. $2 \mathrm{~b}$ CBCT of the frontal region, that shows a thickened palatine mucosa, as well as the presence of $X$-ray positive mass in the cystic cavity 
The macroscopic examination showed a round soft tissue(radicular cyst) with a smooth surface and after the lesion was sectioned a bone sequestrum with hard consistency was observed (fig.3). Pathohistological examination confirmed the diagnosis radicular cyst with bone sequestrum.

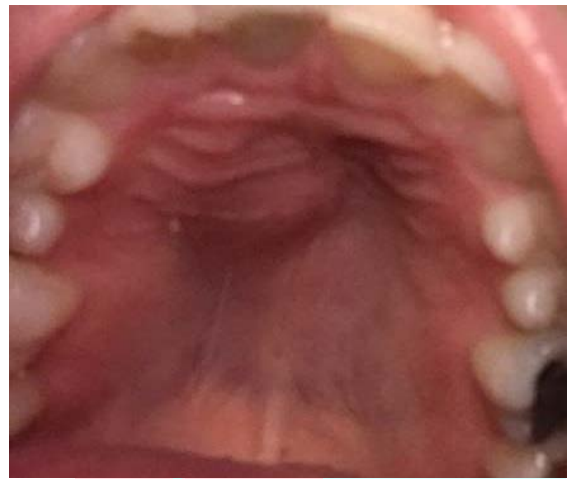

Fig.3 Intraoral view of the formation

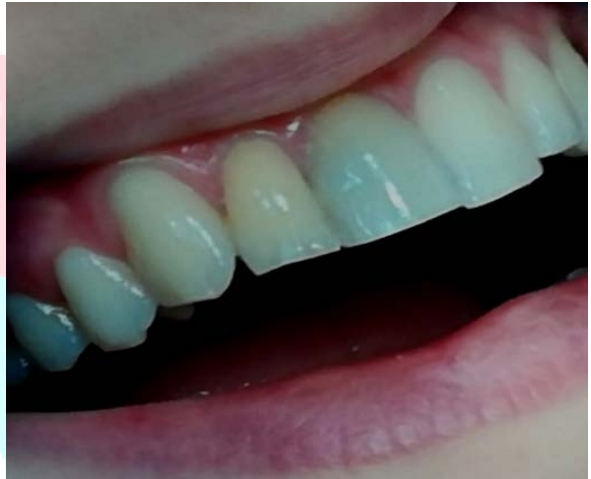

Fig.4 Intraoral view of the discolored teeth

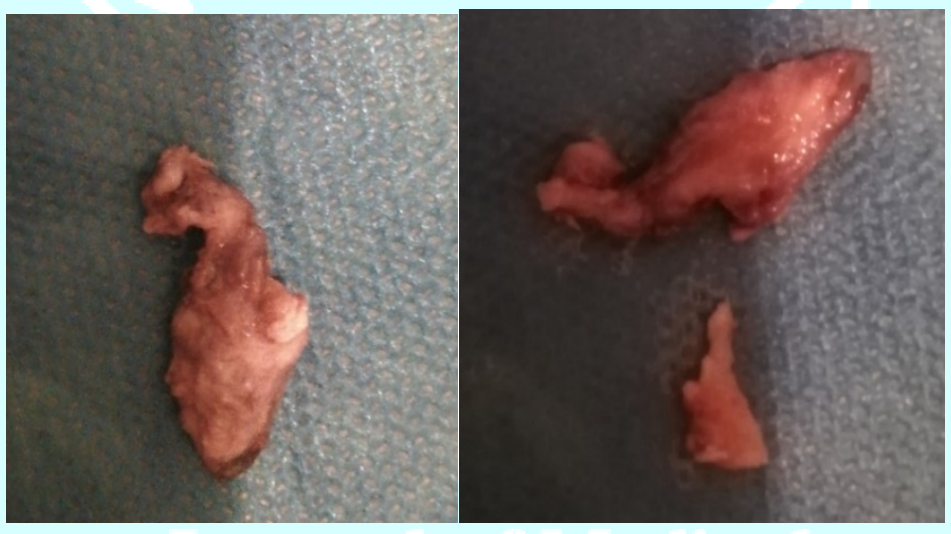

Fig. 5 Macroscopic examination of the formation

Extraction of 12 and enucleation of the lesion was performed and sent for histopathological analysis.(fig. $6,7)$

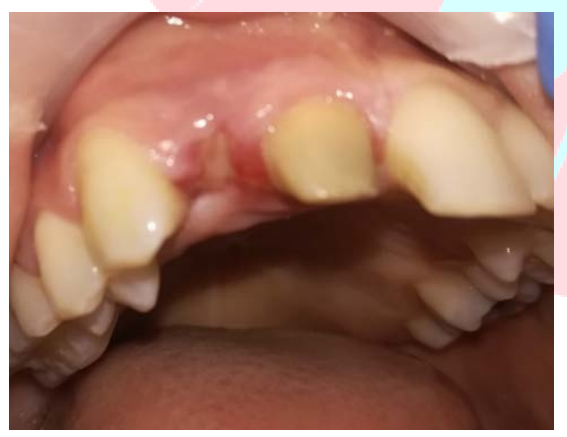

Fig. 6 Extraction ot tooth and enucleation of the lesion

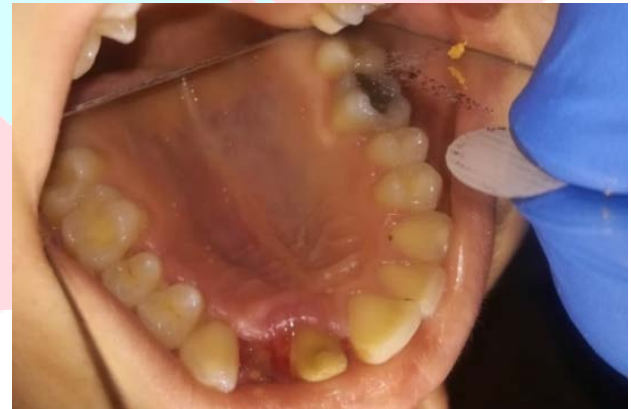

Fig. 7 Intraopearative result 


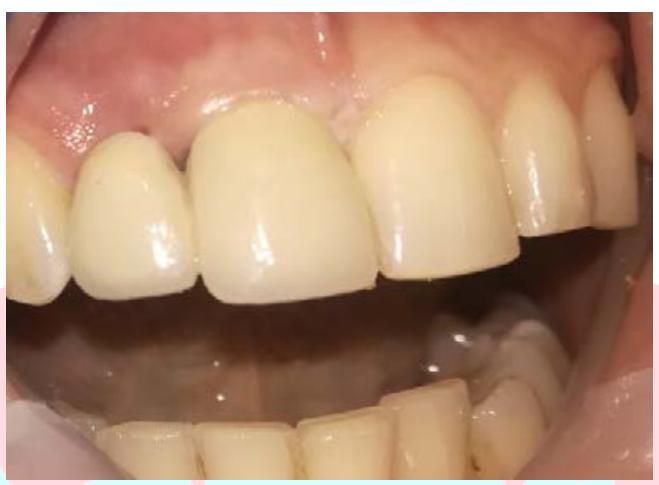

Fig. 8. Postoperative result

15 days after the surgery a temporary construction was placed. (fig. 8)

\section{Discussion}

A radicular cyst is an inflammatory odontogenic cyst of endodontic origin. It is radiologically indistinguishable from a periapical granuloma, except for the size of the lesion. [3] Both lesions are well rounded, unilocular, with a round to oval radiographic image, associated with the apex of the tooth with pulp necrosis or endodontic treatment. They are associated with the destruction of the lamina dura of the affected tooth. The appearance of a radicular cyst as a mixed radiological shadow is a casuistry.

The radicular cyst is formed mainly in the frontal part of the upper jaw, followed by the lateral parts of the upper jaw, the lateral parts of the lower jaw and most rarely in the frontal part of the lower jaw.[4]

Radicular cysts grow slowly and can lead to mobility, root resorption and displacement of adjacent teeth. Radicular cysts could undergo asymptomatic evolution to significant sizes. Depending on the anatomical area in which they develop, the type and degree of clinical symptoms develop is different - swelling, dislocation of adjacent teeth, different degrees of sensory disturbances, crepitation, followed by erosion and fluctuation of the overlying soft tissues. Once infected, the cyst can cause pain and swelling, which alerts patients of an existing problem. [5]

In the case described above, no mobility and displacement of the involved teeth were observed. Radiologically, the resorption of the roots of the upper right central and lateral incisors was visible (apical osteotomy has not been performed). Swelling in the palatal area is present, but the subjective symptom of pain was absent.

A radicular cyst is considered a lesion that develops usually from a dental granuloma. As a result of infectious irritation, epithelial cell rests of Malassez proliferate within the granulation tissue. The epithelial cell rests degenerate, necrotize and liquefaction occurs [1]. A cystic cavity is formed, filled with fluid contents and lined with multilayered squamous epithelium. In the presented case, in addition to the liquid contents, a formation with bone density and structure is detected histologically and macroscopically in the cyst cavity. The available mass is made up of bone structures with signs of degeneration.[6 ]Conventional radiographic image showed a nonspecific and uncharacteristic of a radicular cyst image resembling chronic diffuse periodontitis. Conducting a computed tomography examination makes it possible to detect a thickened palatine mucosa, as well as the presence of X-ray positive mass in the cystic cavity.

Microscopically, the radicular cyst presents a cystic cavity usually lined by non-keratinized stratified squamous epithelium. The epithelial thickness varies depending on the stage of the lesion and the inflammatory reaction level in the fibrous capsule. Dystrophic calcifications may be observed in approximately $15 \%$ of these lesions. A study evaluated a series of residual cyst formations and revealed 
that mineralization increases with time but the amount of calcifications usually does not reflect in mixed radiographic image. But in our case it was not dystrophic calcification but bone sequestrum. It was considered several theories for its formation including chronic inflammation, hypochlorite exposure inside the lesion during endo treatment, trauma, etc.

Several diseases of the jaws may appear as a well-delimited mixed radiographic image. Ossifying fibroma, adenomatoid odontogenic tumor, calcifying cystic odontogenic tumor, calcifying epithelial odontogenic tumor and osseous dysplasia should be considered as a differential diagnosis.[7,8,9]

The treatment of chronic periapical lesions is endodontic treatment and follow-up the patient until complete recovery of the bone lesion. $[3,10,11]$ However, there are cases of lesions that do not respond to conventional endodontic treatment and persist despite it being well performed[12,13]. In these cases it is necessary to carry out a surgical treatment -enucleation of the periapical granuloma/cystic formation and provision of the samples for histological examination, as was done in the case described above.

\section{Conclusion}

Although unilocular radiolucency in periapical X-ray finding is a typical image of radiographic presentation of a radicular cyst, these lesions should be present in the differential diagnostic plan when detecting mixed radiographic images localized in the periapical region of teeth with pulp necrosis or ones that have undergone endodontic treatment. Patients with periapical lesions undergoing conventional endodontic treatment should be monitored until complete clinically and radiologically recovery.

\section{References}

1. Ramos-Perez, Flávia Maria de Moraes, Pontual, Andréa dos Anjos, França, Talita Ribeiro Tenório de, Pontual, Maria Luiza dos Anjos, Beltrão, Ricardo Villar, \& Perez, Danyel Elias da Cruz. (2014). Mixed Periapical Lesion: An Atypical Radicular Cyst with Extensive Calcifications. Brazilian Dental Journal, 25(5), 447-450

2. Lin LM, Ricucci D, Lin J, Rosenberg PA. Nonsurgical root canal therapy of large cyst-like inflammatory periapical lesions and inflammatory apical cysts. J Endod 2009;35:607-615.

3. Shear M. Cysts of the oral and maxillofacial regions. 3rd edn Boston: Wright, 1992

4. Irfan M, Alauddin M, Roselinda A, et al. Big radicular cyst in a 12 year old girl: a case report. Int Med J 2007;6:C5

5. Pekiner FZ, Borahan O, Ugurlu F, et al. Clinical and radiological features of a large radicular cyst involving the entire maxillary sinus. MUSBED 2012;2:31-6

6. Andrade M, Silva-Sousa YT, Marques MF, Pontual ML, Ramos-Perez FM, Perez DE. Ossifying fibroma of the jaws: a clinicopathological case series study. Braz Dent $\mathrm{J}$ 2013;24:662-666.

7. de Noronha Santos Netto J, Machado Cerri J, Miranda AM, Pires FR. Benign fibro-osseous lesions: clinicopathologic features from 143 cases diagnosed in an oral diagnosis setting. Oral Surg Oral Med Oral Pathol Oral Radiol 2013;115:e56-e65.

8. Matos FR, Nonaka CF, Pinto LP, Souza LB, Almeida Freitas R. Adenomatoid odontogenic tumor: retrospective study of 15 cases with emphasis on histopathologic features. Head Neck Pathol 2012;6:430-437.

9. Ortega A, Fariña V, Gallardo A, Espinoza I, Acosta S. Nonendodontic periapical lesions: a retrospective study in Chile. Int Endod J 2007;40:386-390. 
10. Simon JHS. Incidence of periapical cysts in relation to the root canal. J Endod 1980;6:8458

11. Nair PNR. Non-microbial etiology: periapical cysts sustain post-treatment apical periodontitis. Endod Top 2003;6:96-113

12. Heleia NZ, Ene M. Endoscopically assisted enucleation of a large mandibular periapical cyst. Stomatogija, Baltic Dent Maxillofacial J 2011;13:128-31

13. Ribeiro PD, Goncalves ES, Neto ES, et al. Surgical approaches of extensive periapical cyst. Consideration about surgical technique. Salusvita, Bauru 2004;23:317-28

\section{Corresponding author:}

Elitsa Deliverska,

Faculty of Dental Medicine,

Medical University of Sofia,

Department of Dental, Oral and Maxillofacial Surgery,

Sofia 1431 Str. "Georgi Sofiiski 1" 